Musées, Patrimoine et Culture scientifiques et techniques

$132 \mid 2010$

novembre - décembre 2010

\title{
De l'utilité du « texte enfant » au musée
}

The usefulness of 'child-targeted texts' in museums

\section{Amandine Duclos, Franck Philippeaux et Marie-Sylvie Poli}

URL : http://journals.openedition.org/ocim/386

DOI : 10.4000/ocim.386

ISSN : 2108-646X

Éditeur

OCIM

Édition imprimée

Date de publication : 1 novembre 2010

Pagination : 28-33

ISSN : 0994-1908

Référence électronique

Amandine Duclos, Franck Philippeaux et Marie-Sylvie Poli, « De l'utilité du « texte enfant » au musée », La Lettre de I'OCIM [En ligne], 132 | 2010, mis en ligne le 01 novembre 2012, consulté le 01 mai 2019. URL : http://journals.openedition.org/ocim/386 ; DOI : 10.4000/ocim.386

Ce document a été généré automatiquement le 1 mai 2019.

Tous droits réservés 


\title{
De l'utilité du « texte enfant » au musée
}

\author{
The usefulness of 'child-targeted texts' in museums
}

\section{Amandine Duclos, Franck Philippeaux et Marie-Sylvie Poli}

1 Nous appellerons ici «texte enfant » le dispositif de textes (souvent texte/image) destiné aux jeunes visiteurs âgés de huit à douze ans environ. Le choix de cette tranche d'âge s'explique par le fait que les enfants sont alors déjà des lecteurs mais que, éprouvant encore quelques difficultés à lire les textes principaux des musées, ils manifestent aussi leur intérêt pour des textes à première vue simples et concis.

2 Longtemps, le "texte enfant» a été méprisé et ses qualités muséographiques sousestimées (Chauveau, Rogovas-Chauveau, 1996). On lui reprochait notamment de n'être qu'une répétition affaiblie du texte adulte. On pensait alors que l'adulte pouvait tout à fait « traduire » le texte à l'enfant qu'il accompagnait. On verra qu'il y a du vrai dans ce point de vue. Il faudra attendre les années 1990 pour que des chercheurs parviennent à redorer le blason de ce dispositif longtemps négligé. Aujourd'hui, les initiatives pour proposer du texte enfant se multiplient (trop?) dans les musées et de nombreuses institutions l'intègrent désormais à leurs expositions, faisant de lui un dispositif de médiation à part entière. Mais les musées prennent-ils pour autant le temps d'en tester la validité en termes d'autonomie de la visite auprès des principaux intéressés?

3 Les musées de sciences et les musées des beaux-arts ont été parmi les premiers à utiliser ce type de dispositif spécifique et ils sont maintenant rejoints par les musées de société. C'est le cas du musée Dauphinois de Grenoble qui, en 2006 a créé un parcours enfant destiné à des huit-douze ans, dans son exposition ethnographique emblématique Gens de l'alpe. En 2008/2009, une étude qualitative a été menée dans cette exposition pour d'une part, tenter de mieux comprendre comment les huit-douze ans utilisent le texte en général et le texte enfant en particulier et pour, d'autre part, mieux saisir comment le texte écrit permet à ces enfants accompagnés d'adultes (famille), de construire une relation à la fois sensible et cognitive avec les objets exposés dans un musée de société. 


\section{Le « texte enfant » dans Gens de l'alpe au musée Dauphinois : un dispositif que l'équipe fait évoluer pour l'améliorer}

4 Le musée Dauphinois est un musée de société créé en 1906 par l'ethnologue Hippolyte Müller. Cette institution située dans un ancien couvent sur les hauteurs de Grenoble propose des expositions de longue durée ${ }^{1}$ ainsi que des expositions temporaires dont les thématiques et les scénographies sont toujours de qualité. En 1998, le musée Dauphinois inaugure Gens de l'alpe, une exposition de longue durée qui présente la vie des montagnards du XVIIe au XIXe siècle. Cette exposition propose une réflexion sur les rapports entre l'homme, l'animal et la nature tout en insistant sur la nécessité d'une vie collective organisée et d'une ouverture au monde. De nombreux documents, des objets, des mobiliers mais aussi des photographies provenant en grande partie du Queyras (Hautes-Alpes), viennent illustrer ce propos, dont le texte écrit.

5 En 2006, à l'occasion du centenaire du musée Dauphinois, le conservateur du musée et concepteur de Gens de l'alpe ${ }^{2}$, Jean-Claude Duclos, souhaite enrichir l'exposition et la rendre plus accessible aux jeunes visiteurs. Précisons que Gens de l'alpe est une exposition pédagogique plébiscitée par les publics individuels mais aussi par les familles et les groupes scolaires. Le conservateur opte alors pour la création d'un dispositif scénographique spécialement conçu pour les enfants de huit à douze ans.

6 La scénographe Anne Surot réalise un premier parcours enfant composé de treize textes colorés, présentés dans des caissons lumineux rétro éclairés et placés à proximité des textes adultes. Leurs énoncés sont simples et gaiement ornés à la manière des livres de contes pour enfants. Chacun de ces textes aborde un thème particulier en lien avec les grandes thématiques de l'exposition. En plus des textes, Gens de l'alpe propose dès lors des éléments mobiliers spécifiquement pensés pour les jeunes visiteurs comme des carillons de sonnailles, des trappes à ouvrir, un rouleau des saisons à faire tourner, des éléments décoratifs, le tout pour permettre - en principe - aux enfants de se familiariser tactilement avec les sujets abordés.

7 Une étude à visée évaluative de l'ensemble de ce dispositif menée en 2007 permet de déceler certains dysfonctionnements en ce qui concerne surtout l'attractivité des textes pour les huit-douze ans. Ainsi par exemple, alors que l'équipe pensait bien faire en optant pour des textes à l'esthétique proche des livres de littérature enfantine classique (typographie stylisée, croquis naïfs, couleurs acidulées), il s'avère que les jeunes visiteurs négligent ces textes qu'ils jugent « trop bébé ». Par ailleurs, l'étude par observation et par entretien montre également que dès le premier tiers du parcours de l'exposition, les enfants cessent de prêter attention aux textes enfants qui leur sont pourtant spécifiquement destinés. Fort de ces résultats, le musée Dauphinois décide de modifier à nouveau son approche des textes enfants. L'idée étant cette fois de mettre en place des textes informatifs courts relevant surtout du registre injonctif (qui pousse le lecteur à faire quelque chose) afin que l'enfant soit verbalement et iconographiquement incité à observer, dans son périmètre visuel, une sélection d'objets issus des collections.

8 Dans cette seconde tentative qui fait partie intégrante de l'étude de réception dont nous rendons compte ici, les textes enfants sont toujours présentés dans des caissons lumineux rétro éclairés mais la ressemblance avec l'ancien dispositif s'arrête là. En effet, les 
illustrations ne s'inspirent plus de l'univers du conte enfantin. Le style des cartels pour enfants opte pour un contraste blanc/noir/oranger, la typographie et les formes dessinées sont très modernes, très graphiques. Tous les signes de référence sont puisés dans l'exposition elle-même (objets, vidéos, textes). Les illustrations graphiques des énoncés représentent des expôts emblématiques de Gens de l'alpe comme le coffin ${ }^{3}$, la herse ou les crampons d'alpinisme, par exemple.

Certes, ces textes pour enfant conservent une dimension pédagogique indispensable à la bonne compréhension des histoires et des fonctions sociales des objets présentés. Ainsi les objets sont-ils nommés et leurs fonctions techniques ou sociales précisées en quelques mots adéquats. Chaque fois que cela est possible, un énoncé prescriptif du style « Regarde, il y a une herse derrière toi ! » permet de faire correspondre un objet de collection à un terme (vocabulaire technique) précis, l'objectif étant de motiver le jeune visiteur pour qu'il s'intéresse aux objets présentés. Car pour le concepteur de l'exposition, ce sont avant tout les objets qui doivent permettre à l'enfant de saisir la dimension culturelle (anthropologique) du parcours de l'exposition. On l'aura compris, dans Gens de l'alpe, l'objet est placé au centre de l'exposition, il constitue le témoin privilégié des modes de vie passés des habitants de l'alpe.

\section{Une attention particulière portée à la relation que l'enfant instaure avec tous les types de texte au cours de sa visite}

10 En 2009, une troisième étude sur l'usage du texte enfant par les huit-douze ans dans Gens de l'alpe est amorcée à partir de ce nouveau dispositif ${ }^{4}$. Pour mener cette étude, nous avons choisi de procéder à une évaluation formative (Taylor, 1998), à savoir évaluer les manières dont les visiteurs utilisent les dispositifs existants. Il ne s'agit donc pas de comparer cette version des textes au dispositif enfant précédent.

11 L'objectif premier de cette étude consiste à tenter de comprendre comment l'enfant va se comporter en tant que lecteur et en tant que visiteur, seul et avec les personnes qui l'accompagnent, dans une exposition dotée d'un registre de textes à vocation prescriptive, des textes de médiation précisément destinés à amener les enfants à voir/ examiner les objets. Du point de vue méthodologique, nous devons avouer qu'il n'a pas été aisé, sur la durée de l'évaluation (quatre semaines dont les week-ends), de trouver beaucoup de groupes de visiteurs composés d'un ou de deux enfants venus avec un ou des adultes, qui acceptent de s'engager dans le dispositif d'enquête relativement contraignant que nous proposions (prendre des photographies au cours de la visite et ensuite répondre à nos questions, sans faire participer les adultes accompagnateurs à l'interview).

12 Au final nous avons mené dix entretiens semi directifs avec six filles et quatre garçons âgés de huit-douze ans venus en famille en dehors du cadre scolaire, en semaine ou le week-end. L'entretien avec les enfants comportait vingt trois questions centrées sur leur perception des dispositifs enfants et sur l'usage qu'ils déclaraient avoir fait des textes écrits au cours de leur visite. Comme nous les y avions invités, certains ont pris une dizaine de photographies pendant leur visite (la consigne étant : « photographie ce que tu as envie »). Les photographies servaient de point de départ à la discussion et permettaient aux enfants de s'exprimer en se référant à des témoins visuels concrets de leur expérience de visite, de leurs goûts aussi. 
13 Par ailleurs, comme nous souhaitions aussi nous concentrer sur le comportement de découverte des visiteurs et sur les éventuelles interactions pouvant s'établir entre adultes et enfants au cours de la visite, nous avons également mené des observations à l'insu des jeunes visiteurs et des adultes qui les accompagnaient (parents, grands-parents). En effet, cette méthode d'étude par observation permet d'être dans l'exposition au plus près des visiteurs, sans que ceux-ci ne s'en aperçoivent. Ainsi ne modifient-ils pas leurs pratiques de visite et le recueil de données se déroule de manière satisfaisante.

\section{Un résultat intéressant : les huit-douze ans se comportent déjà en visiteurs-lecteurs}

Le premier résultat intéressant de cette étude concerne l'attitude que les enfants ont développée de manière quasi spontanée devant le médium texte, qu'il soit spécifiquement conçu pour les jeunes visiteurs ou pas. Sur les dix enfants interrogés et les dix observés, tous ont eu recours au média textuel durant leur visite. Et même si leurs pratiques de lecture sont parcellaires et hétérogènes, ce recours aux cartels adultes et aux textes enfants témoigne de l'importance que revêt pour eux le média textuel au musée. Qui plus est, les enfants interrogés ont été à même de justifier leur pratique, comme par exemple : «Ben, je voulais savoir ce que c'était que les objets que je voyais alors j'ai lu le petit texte carré et comme y'avait pas assez d'informations, alors j'ai lu le grand texte » (fille, 10 ans, venue avec son grand-père et son frère).

\section{Mais lire, ce n'est pas toujours bien comprendre le propos de l'exposition ou la fonction de l'objet}

Nous nous sommes aperçus lors des entretiens que même s'ils lisaient les textes enfants et certains textes adultes, les enfants ne saisissaient pas correctement les repères historiques et ethnographiques du discours de l'exposition: "On nous parle de la Préhistoire, comment vivaient les hommes avant...» (garçon, 10 ans, venu avec son grand-père et sa sœur).

Autre problème, le sujet précis d'un texte peut être déchiffré et mal compris : «J'ai lu le texte et c'était une bonne manière pour protéger les moutons. Les loups pouvaient pas les manger parce qu'il y avait des épines pour les piquer » (fille, 8 ans et demi, venue avec son père). Ici la fillette a lu le texte mais n'en a pas compris le contenu. Le texte explique que les colliers exposés servent à protéger les chiens de berger, non pas les moutons.

Précisons néanmoins que, quelques erreurs mises à part, les enfants interrogés ont été capables de résumer l'exposition, preuve qu'ils en ont compris les significations d'ensemble. Ils ont retenu les principales notions abordées comme celles d'habitat, de vie avec les animaux, ou de travail de la terre.

Par contre, la notion de vie partagée et de nécessaire entraide entre familles pour lutter contre les conditions climatiques difficiles ne semble pas avoir été intégrée. Est-ce une question d'âge? Est-ce parce que nos jeunes interviewés étaient des citadins? On peut faire cette double hypothèse. 
19 Sur les dix enfants interrogés, il n'est donc pas possible de dire avec certitude que la lecture du texte enfant vérifiée par l'observation a favorisé la compréhension approfondie des messages des concepteurs.

\section{On peut s'amuser et lire pour comprendre en même temps... Si on est déjà lecteur}

20 Lors des entretiens, nous cherchions à savoir si l'expérience de visite des enfants visiteurs de Gens de l'alpe comportait ou non un côté ludique. Les réponses des dix ont montré que oui. Par ailleurs, l'analyse détaillée des réponses établit de manière certaine un lien entre le sentiment déclaré de s'être amusé et le fait d'avoir cherché à répondre aux « questionsjeux » des textes enfants, des questions en relation directe avec des objets exposés.

21 Ainsi, M. (fille, 8 ans et demi, venue avec sa mère et son frère) explique s'être « amusée en répondant aux questions orange dans les petits carrés orange ", à savoir les questions des textes enfants. Cette petite fille a, notons le, comme les autres enfants qui ont joué avec les textes enfants, très bien saisi le message des textes enfants et le propos ethnographique global de l'exposition.

Ce résultat s'inscrit en faveur de ces dispositifs écrits qui tablent sur la fonction ludique du rapport à l'exposition, rapport qui passe aussi par le texte lu. Or, c'est le point de recoupement entre les trois résultats évoqués jusqu'à présent: les enfants observés et interrogés dans Gens de l'alpe possédaient un «habitus de lecture " riche et varié leur permettant d'être à l'aise avec l'écrit au musée. C'est du moins ce qu'on peut induire des réponses qu'ils ont fournies à nos questions sur leurs relations à la lecture, les dix ayant déclaré aimer lire des histoires, des bandes dessinées, des petits romans...

Qu'en est-il pour les enfants dont les pratiques familiales ne permettent pas cette familiarité avec l'écrit, avec la lecture ? Pour autant, nous savons bien que c'est la société toute entière qui pousse un enfant à lire (dans la rue, dans les transports, les magasins...) et pas seulement ses parents; ce qui explique qu'au musée comme ailleurs, les jeunes visiteurs ont tendance à utiliser le média textuel pour donner du sens au contexte de communication dans lequel ils sont placés.

\section{De l'adulte à l'enfant : une relation plutôt pédagogique, avec peu de lecture partagée}

$\mathrm{Au}$ commencement de notre recherche, nous avons émis les deux hypothèses suivantes à propos des relations entre enfants et adultes vis-à-vis du texte enfant dans Gens de l'alpe. Premièrement, nous avons fait l'hypothèse que l'adulte chercherait à transmettre des connaissances à l'enfant qui l'accompagnait, établissant alors avec lui une relation que nous pourrions qualifier de "scolaire ". Seconde hypothèse, nous pensions que cette transmission de savoir n'était que le point de départ d'une relation approfondie, composée d'interactions complices entre l'enfant et l'adulte. Cette conception de la visite familiale « idéale », sans doute un peu naïve, allait bientôt être ébranlée par la réalité du terrain.

De fait, nos observations nous ont révélé que lors de la visite de Gens de l'alpe, l'adulte (père, mère, grands-parents) était entièrement tourné vers l'enfant. En effet, l'adulte 
cherchait à capter son attention, attiser sa curiosité et surtout, il cherchait à transmettre des connaissances à l'enfant. Pour ce faire, l'adulte posait des questions à l'enfant, il l'invitait à regarder tel ou tel expôt et lui donnait verbalement et gestuellement de nombreuses informations, sans avoir pour autant pris la peine de lire les textes informatifs auparavant (enfant ou tout public).

De plus, dans les groupes observés, l'adulte imposait un ordre ainsi qu'un rythme de visite au jeune visiteur, décidait des espaces où il convenait de s'arrêter et du temps qu'il convenait d'y passer. Dans les dix groupes observés, l'adulte était d'abord soucieux de transmettre ses propres connaissances à l'enfant, utilisant pour cela très peu, voire pas du tout les dispositifs de médiation de l'exposition.

À quelques exceptions près (deux discussions prolongées à propos de la râpe à pain ${ }^{5}$ entre adultes et enfants à partir du texte enfant), nos hypothèses n'ont pas été validées : les textes enfants n'ont pas fonctionné comme des outils de médiation à part entière au sujet desquels les visiteurs adultes et les visiteurs enfants ont discuté, ont échangé. Durant notre étude, les textes pour les adultes se sont révélés plus propices à des échanges enfants/adultes.

\section{Conclusion}

Du point de vue des seuls résultats traités, cette étude de terrain consacrée aux usages que les jeunes visiteurs et leurs familles font des dispositifs de texte enfant dans une exposition comme Gens de l'alpe, nous a permis de constater premièrement que les enfants observés et interrogés nous ont convaincus de la nécessité pour un musée à vocation pédagogique comme le musée Dauphinois, de proposer des dispositifs de textes prescriptifs pour jeunes visiteurs qui "parlent» avec rigueur et précision mais sans jargonner, des objets mis en valeur par l'écriture scénographique. Par contre, nous n'avons pas validé l'efficacité d'une dimension ludique des textes enfants sur l'ensemble du parcours.

Deuxièmement, alors que nous avions fait l'hypothèse que les adultes qui accompagnaient les jeunes visiteurs se saisiraient des textes enfants pour engager avec eux des échanges sur les contenus de ces textes, nous avons constaté que les dispositifs que nous avions conçus à cet effet n'ont pas permis d'atteindre cet objectif.

Enfin, cette étude confirme qu'en prenant pour objet de réflexion le rôle de médiateur que peut tenir le «texte enfant» lors d'une visite en famille, on fait émerger d'autres questionnements muséologiques de première importance comme les représentations pédagogiques du média exposition chez les adultes accompagnants ou les pratiques de lecture et de captation des informations des adultes dans l'exposition lorsqu'ils se donnent eux-mêmes la mission « d'enseigner » l'enfant qui les accompagne.

Ces questions méritent de faire l'objet de futures études centrées sur les relations adultes/enfants. Ce ne sera possible que si d'autres musées, comme le musée Dauphinois, s'investissement régulièrement dans une démarche de recherche évaluation. 


\section{BIBLIOGRAPHIE}

Chauveau, G. et Rogovas-Chauveau, E. Les jeunes enfants et l'entrée dans l'écrit, la Lettre de l'OCIM $\mathrm{n}^{\circ} 43,1996$.

Duclos, A. Réception du texte enfant dans l'exposition « Gens de l'alpe ». Rapport d'étude, musée Dauphinois, Grenoble, 2009.

Guichard, J. Adapter la muséologie aux enfants, in Schiele, B. et Koste, E. La Révolution dans la muséologie des sciences. Presses Universitaires de Lyon et Éditions MultiMondes, 1998.

Nazor, O. Réflexions sur la notion de musées, in Mairesse, F. et Desvallées, A. Vers une redéfinition du musée ? Paris : Éditions L’Harmattan, 2007.

Taylor, S. Essayer-Modifier. Comment améliorer des éléments d'exposition avec l'évaluation formative. Traduction et adaptation de l'édition française par Daniel Jacobi et Joëlle Le Marec. OCIM, 1998.

\section{NOTES}

1. Une exposition de longue durée est une exposition qui peut bénéficier de remaniements scénographiques réguliers plus ou moins importants, l'objectif étant de tenir compte de l'avancée des connaissances sur le sujet ou bien de compléter la présentation de l'exposition par de nouveaux médias ou de nouveaux expôts.

2. La présentation complète de l'exposition Gens de l'alpe est disponible sur le site du musée Dauphinois www.musee-dauphinois.fr

3. Le coffin était un étui que le faucheur portait à la ceinture. Il servait à ranger la pierre à aiguiser la faux.

4. L'étude est réalisée par Amandine Duclos lors de son stage de M1 au service des publics du musée Dauphinois.

5. Une râpe à pain était un ustensile permettant d'émietter le pain dur afin de le mélanger à un liquide comme du lait, de la soupe ou du bouillon

\section{RÉSUMÉS}

Il ne s'agit pas ici de donner des « recettes » à propos des écrits d'aide à la visite destinés au public enfant en général mais plutôt de livrer quelques pistes de réflexion et de revenir sur certains clichés à propos du "texte enfant » dans les musées de société en s'appuyant sur une étude menée au musée Dauphinois de Grenoble sur les écrits informatifs destinés aux huit-douze ans dans l'exposition « Gens de l'alpe ».

It is not a question in this article of giving 'recipes' on helpful texts aimed at child-visitors, but rather to give a few avenues of exploration and to come back on certain clichés about 'child- 
targeted texts' in museums. This exploration is based on a study on informative texts aimed at eight to twelve year olds in the 'People of the Alps' exhibition that took place in the Dauphinois museum in Grenoble.

INDEX

Mots-clés : exposition, jeune public, médiation, musée Dauphinois, texte, enfants Index géographique : France, Grenoble

\section{AUTEURS}

AMANDINE DUCLOS

Amandine Duclos est étudiante en Master 2 Art et Culture de l'université Pierre Mendès France de Grenoble

\section{FRANCK PHILIPPEAUX}

Franck Philippeaux est chargé de la médiation culturelle au musée dauphinois.

f.philippeaux@cg38.fr

\section{MARIE-SYLVIE POLI}

Marie-Sylvie Poli est professeur à l'université Pierre Mendès France de Grenoble, équipe Culture et Communication, centre Norbert Élias, université d'Avignon et des Pays du Vaucluse. marie-sylvie.poli@upmf-grenoble.fr 